Draft VERSion July 21, 2021

Typeset using $\mathrm{LAT}_{\mathrm{E}} \mathrm{X}$ twocolumn style in AASTeX63

\title{
Revisiting FUSE O VI Emission in Galaxy Halos
}

\author{
Haeun Chung, ${ }^{1}$ Carlos J. Vargas, ${ }^{1}$ And Erika Hamden ${ }^{1}$ \\ ${ }^{1}$ University of Arizona, Steward Observatory, 933 N. Cherry Ave., Tucson, AZ 85721, USA
}

(Dated: January 2021; Received; Revised; Accepted)

\begin{abstract}
A significant fraction of baryons in galaxies are in the form of diffuse gas of the circumgalactic medium (CGM). One critical component of the multi-phases of CGM, the so-called "coronal" warm-hot phase gas $\left(10^{5}-10^{6} \mathrm{~K}\right)$ traced by O VI 1031.93, $1037.62 \AA$ resonance lines, has rarely been detected in emission from galaxy halos other than Milky Way. Here we report four additional detections of O VI emission gas in the halos of nearby edge-on galaxies, NGC 4631 and NGC 891, using archival Far Ultraviolet Spectroscopic Explorer data and an updated data pipeline. We find the most intense O VI emission to be from fields forming a vertical line near the center of NGC 4631, despite the close proximity to the disk of two other fields. The detected O VI emission surface brightness are about $1.1 \pm 0.3 \times 10^{-18}$ to $3.9 \pm 0.8 \times 10^{-18} \mathrm{ergs} \mathrm{s}^{-1} \mathrm{~cm}^{-2} \operatorname{arcsec}^{-2}$. The spatial distribution of the five $30^{\prime \prime} \times 30^{\prime \prime} \mathrm{O}$ VI detection fields in NGC 4631 can be interpreted as the existence of filamentary structures of more intense O VI emission superimposed within a diffuse and faint O VI halo in star-forming galaxies. Volume-filled O VI emission mapping is greatly needed to determine the structure and prevalence of warm-hot gas and the role it plays in the cycling of gas between the galaxy disk and the halo. Finally, we present the sensitivity of future funded and proposed UV missions (LUVOIR-A, LUVOIR-B, CETUS, and Aspera) to the detection of diffuse and faint O VI emission in nearby galaxy halos.
\end{abstract}

\section{INTRODUCTION}

In the Lambda Cold Dark Matter $(\Lambda \mathrm{CDM})$ paradigm, galaxies form at the centers of dark matter halos out of cooling and condensed gas into a central star-forming disk. While observations of the galaxies at the center of these halos have been conducted for over 100 years, we are only now able to understand the full picture of how gas outside of the central disk plays a key role in the evolution and star formation rate of the galaxy. This full picture of gas is important because a majority $(>80 \%)$ of the gas in most galaxies will never end up in stars (Zaritsky \& Courtois 2017; Tumlinson et al. 2017; Behroozi et al. 2019). In these halos, the warmhot $\left(10^{5}-10^{6} \mathrm{~K}\right)$ phase of the CGM accounts for more mass than the stars within the parent galaxy. This phase is best traced by the $\mathrm{O}$ VI doublet, occurring at $\lambda \lambda 1031.93,1037.62 \AA$ rest frame. This transition is extremely temperature sensitive, and is the strongest line transition that traces warm-hot phase gas. Emission

Corresponding author: Haeun Chung

haeunchung@arizona.edu from even this brightest transition occurs at low surface brightness which has challenged observers.

Due to the historical difficulty of measuring O VI emission, only three studies have presented detections of coronal O VI emission lines outside of the Milky Way: two in galaxy disks and one in the CGM (Otte et al. 2003; Grimes et al. 2007; Hayes et al. 2016). Otte et al. (2003)(hereafter O03) provides the most directly relevant results to this work. They searched for O VI emission beyond the disks of two nearby edge-on galaxiesNGC 4631 and NGC 891-using Far Ultraviolet Spectroscopic Explorer (FUSE, Moos et al. 2000) spectra. They detected coronal gas in two fields $\left(30^{\prime \prime} \times 30^{\prime \prime}\right.$ Fieldof-view) of NGC 4631 and provided upper limits for NGC 891 (See Figure 1).

NGC 4631, also known as the Whale Galaxy, is a nearby edge-on barred spiral galaxy with companions NGC 4627 and NGC 4656 (Richter et al. 2018). NGC 4631 has a distance of $7.4 \pm 0.2 \mathrm{Mpc}$, and, based on $\mathrm{H}$ I data, is interacting with several nearby systems (Radburn-Smith et al. 2011). NGC 4631 was originally observed with FUSE because ROSAT (Pfeffermann et al. 1987) observations indicated a concentration of soft X-ray emission above the plane of the galaxy but correlated with star formation activity in the plane 
Table 1. FUSE Observation Summary

\begin{tabular}{ccccccc}
\hline \hline Field & Program ID & $\begin{array}{c}\text { R.A. } \\
(\mathrm{J} 2000)\end{array}$ & $\begin{array}{c}\text { Decl. } \\
(\mathrm{J} 2000)\end{array}$ & $\begin{array}{c}\text { Exposure Time } \\
\text { (Day+Night, sec })\end{array}$ & $\begin{array}{c}\text { Exposure Time } \\
\text { (Night, sec })\end{array}$ & $\begin{array}{c}\text { Effective Area } \\
\left(\text { LiF1-A, } \mathrm{cm}^{2}\right)\end{array}$ \\
\hline NGC4631-A & p1340101 & $12 \mathrm{~h} 42 \mathrm{~m} 08.8 \mathrm{~s}$ & $+32 \mathrm{~d} 34 \mathrm{~m} 36.0 \mathrm{~s}$ & 21536 & 12737 & 26.9 \\
NGC4631-B & $\mathrm{p} 1340201$ & $12 \mathrm{~h} 42 \mathrm{~m} 08.8 \mathrm{~s}$ & $+32 \mathrm{~d} 33 \mathrm{~m} 36.0 \mathrm{~s}$ & 16218 & 11528 & 26.9 \\
NGC4631-A* & $\mathrm{c} 0570101$ & $12 \mathrm{~h} 42 \mathrm{~m} 08.8 \mathrm{~s}$ & $+32 \mathrm{~d} 34 \mathrm{~m} 36.0 \mathrm{~s}$ & 7153 & 6332 & 24.6 \\
NGC4631-F & $\mathrm{c} 0570201$ & $12 \mathrm{~h} 42 \mathrm{~m} 18.0 \mathrm{~s}$ & $+32 \mathrm{~d} 33 \mathrm{~m} 48.0 \mathrm{~s}$ & 16339 & 13573 & 23.2 \\
NGC4631-F* & $\mathrm{c} 0570202$ & $12 \mathrm{~h} 42 \mathrm{~m} 18.0 \mathrm{~s}$ & $+32 \mathrm{~d} 33 \mathrm{~m} 48.0 \mathrm{~s}$ & 30228 & 18134 & 23.3 \\
NGC4631-H & $\mathrm{c} 0570301$ & $12 \mathrm{~h} 41 \mathrm{~m} 56.0 \mathrm{~s}$ & $+32 \mathrm{~d} 33 \mathrm{~m} 12.0 \mathrm{~s}$ & 22866 & 15856 & 23.4 \\
NGC4631-I & $\mathrm{c} 0570401$ & $12 \mathrm{~h} 42 \mathrm{~m} 10.0 \mathrm{~s}$ & $+32 \mathrm{~d} 33 \mathrm{~m} 06.0 \mathrm{~s}$ & 9392 & 8447 & 24.3 \\
NGC891-1 & $\mathrm{b} 1140101$ & $2 \mathrm{~h} 22 \mathrm{~m} 29.0 \mathrm{~s}$ & $+42 \mathrm{~d} 21 \mathrm{~m} 12.0 \mathrm{~s}$ & 31594 & 27174 & 26.2 \\
NGC891-2 & $\mathrm{b} 1140201$ & $2 \mathrm{~h} 22 \mathrm{~m} 40.0 \mathrm{~s}$ & $+42 \mathrm{~d} 22 \mathrm{~m} 36.0 \mathrm{~s}$ & 31440 & 27203 & 26.3 \\
NGC891-3 & $\mathrm{b} 1140301$ & $2 \mathrm{~h} 22 \mathrm{~m} 44.8 \mathrm{~s}$ & $+42 \mathrm{~d} 22 \mathrm{~m} 12.0 \mathrm{~s}$ & 18485 & 15666 & 26.2 \\
\hline
\end{tabular}

${ }^{a}$ Effective area of FUSE LiF1-A channel at $1035 \AA$.

(Murphy 2002). Additional observations of NGC 891 were conducted later, in part because of similar soft Xray emission detected by ROSAT. NGC 891 is a nearby, edge-on unbarred spiral galaxy at a distance of $9.1 \pm$ 0.4 Mpc (Radburn-Smith et al. 2011), but unlike NGC 4631, does not have any large companions. NGC 891 is usually compared to our own Milky Way galaxy, in both size and luminosity. There have been numerous studies of the halo of NGC 891 (Temple et al. 2005; Hodges-Kluck et al. 2018; Qu et al. 2019; Das et al. 2020), which indicate the presence of both neutral hydrogen, a hot halo, and warm ionized gas of less than solar metallicity. Observations by FUSE of NGC 4631 and NGC 891 were conducted between 2000 and 2004.

Here, we present an updated analysis of the FUSE data presented in O03, including several fields which were not included in their original published study. We detect coronal gas via O VI in several sightlines, three of which are aligned in a possible filamentary structure. In Section 2, we describe the FUSE data and our updated analysis. In Section 3, we describe our detections and limits for NGC 4631 and NGC 891. Finally, we discuss the implications of this new analysis in Section 4, including the impact on future EUV mission concepts.

\section{DATA AND ANALYSIS}

Our analysis uses existing archival data from the FUSE telescope (Moos et al. 2000), which was launched in 1999 and operated for 8 years. The data used in this analysis is listed in Table 1.

\subsection{The FUSE Telescope}

FUSE was designed to observe in the extreme FUV (905-1187 $\AA$ ), and was originally designed to follow up the Copernicus mission (Rogerson et al. 1973) with higher sensitivity and spectral resolution to capitalize on the rich variety of astrophysically important lines in the EUV. FUSE consists of four co-aligned off-axis parabola telescopes (each $352 \times 387 \mathrm{~mm}$ ), which each feed light to four Rowland circle type spectrographs. All four channels observe the same field of view, but two channels are optimized for 905-1105 $\AA$ while the remaining two are optimized for 1000-1187 A. The FUSE focal plane consisted of two microchannel plate (MCP) detectors (Siegmund et al. 1997).

\subsection{New unpublished data}

The data presented here consists of 10 FUSE lowresolution aperture (LWRS) pointings, which include two galaxies (NGC 4631 and NGC 891) and is a mixture of previously published and unpublished data. The data presented here is publicly accessible.

\subsection{Updated analysis of previously published data}

\subsubsection{FUSE data}

The FUSE raw data is downloaded from the Canadian Astronomy Data Centre. The data is further processed by the latest release of CalFUSE (v3.2.3, Dixon et al. 2007). We use the default pulse height range (between 2 and 25, inclusive) of CalFUSE v3.2.3, which is wider than the range used by $\mathrm{O} 03$ (between 4 and 15, inclusive). Using the default pulse height range is strongly advised by the pipeline developers, since the use of narrower pulse height range can lead to a significant flux loss (Dixon et al. 2007). The extracted spectrum from exposures of each observing program ID are combined using FUSE Tools in $\mathrm{C}^{1}$ (idf_combine, cf_bad_pixels, bpm_combine, and cf_extract_spectra), following the recommendation from the web instruction. This is to

\footnotetext{
${ }^{1}$ https://archive.stsci.edu/fuse/analysis/toolbox.html
} 

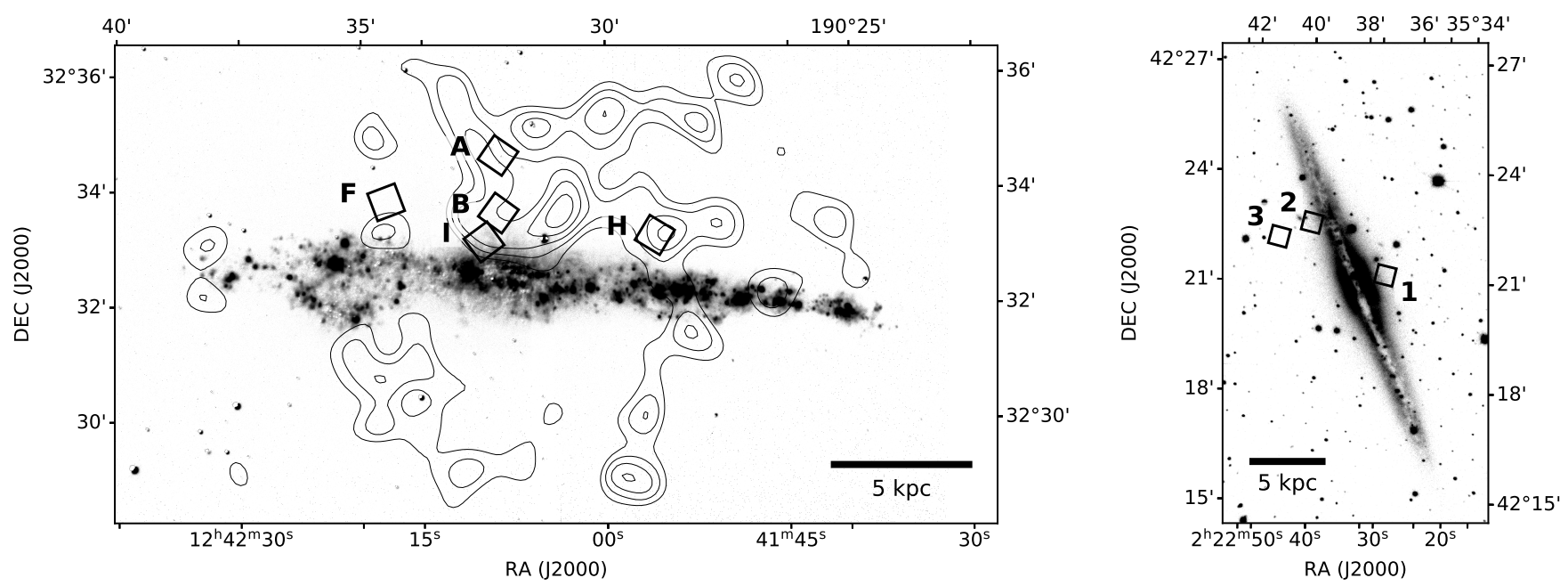

Figure 1. (Left) $\mathrm{H} \alpha$ image of NGC 4631 with soft X-ray band ( $0.15-0.3 \mathrm{keV})$ contours. Five FUSE LWRS observation fields are marked with black squares. NGC 4631-A and NGC 4631-B are previously known O VI detections by O03. H $\alpha$ image is adopted from SINGS data archive (Kennicutt et al. 2003), observed by KPNO 2.1m telescope. Each contour interval equals $1 \sigma$ with the lowest contour at $3 \sigma$ aboave the local sky background $\left(3.8 \times 10^{-4}\right.$ counts s$^{-1}$ arcmin $^{-2} ; 1 \sigma=1.8 \times 10^{-4}$ counts s$^{-1}$ $\operatorname{arcmin}^{-2}$ ). $7.4 \mathrm{Mpc}$ is used as the distance to NGC 4631 (Radburn-Smith et al. 2011). (Right) H $\alpha$ image of NGC 891 with three FUSE LWRS observation fields, marked with black squares. All three fields were previously known as O VI non-detections (O03). The image is adopted from Cheng et al. (1997), observed by Mount Laguana Observatory SDSU 40-inch telescope. 9.1 Mpc is used as the distance to NGC 891 (Radburn-Smith et al. 2011).

best estimate the background level when the targets are faint. Among the output of the pipeline, we use wavelength (WAVE) and the dead-time corrected counts values (WEIGHTS) as a spectrum (Figure 2). The background count spectrum (BKGD) is used to calculate the signal (Signal = WEIGHTS - BKGD). The raw counts spectrum (COUNTS) is also used for generating Poisson random noise added spectrum which is described in the following paragraph.

Before measuring the SNR of the O VI $1032 \AA$ emission, we fitted the O VI $1032 \AA$ line emission by a line profile model to measure its kinematics and determine the optimal extraction window size. The model profile is a convolution of three functions 1) a Gaussian function as a $\mathrm{O}$ VI emission line model, 2) a function representing the FUSE point-spread-function (PSF), assumed to be Gaussian with a Full-Width Half-Maximum $(\mathrm{FWHM})=0.021 \AA$ (corresponds to the spectral resolution at the central wavelength of LiF1-A channel (1034.7 $\AA, \mathrm{R} \sim 21000)$ ), and 3) a top-hat function corresponding to the width of the LWRS slit $\left(30^{\prime \prime}\right)$. Four parameters (Amplitude and the FWHM of the O VI emission model, a center of the convolved function, and a constant) are set as free parameters. For each spectrum, we have generated 1000 Poisson random-noise added spectra (according to the SNR at each spectrum bin). The convolved function is fitted to each noise added spectrum using a non-linear least square fitting method (scipy.optimize.curve_fit). An average and standard deviation of the fitted parameters (line center and FWHM) from 1000 fittings are determined as the parameter value and error. We put an example of this line fitting to NGC 4631-A spectrum in Appendix A. The fitting was not attempted for the spectra with no visible O VI $1032 \AA$ emission line (NGC 891-1 and 3).

After the fitting, we measured SNR of the O VI 1032 $\AA$ emission line. The size of the extraction window (in the wavelength dimension) is determined as the width corresponding to $95 \%$ of the area of the final convolved function. The final convolved function is the convolution of the three functions (two Gaussians and one tophat), adopting the O VI emission FWHM value from the fitting result. The width of the extraction windows are shown in the grey shaded region in Figure 2. The $\mathrm{O}$ VI signal count is calculated by the difference between the total event counts and the total background counts within the extraction window. The O VI signal count is converted to flux per unit angular area in both counts s ${ }^{-1} \mathrm{~cm}^{-2} \mathrm{sr}^{-1}$ (Line Unit, LU) and ergs s ${ }^{-1}$ $\mathrm{cm}^{-2} \operatorname{arcsec}^{-2}$. The FUSE effective area at the time of observation is estimated by the interpolation between the two closest FUSE effective area data to the epoch time of each observation. The time-dependent spectral effective area curves are available from the CalFUSE calibration files. The mean effective area values at $1035 \AA$ are presented at Table 1. 


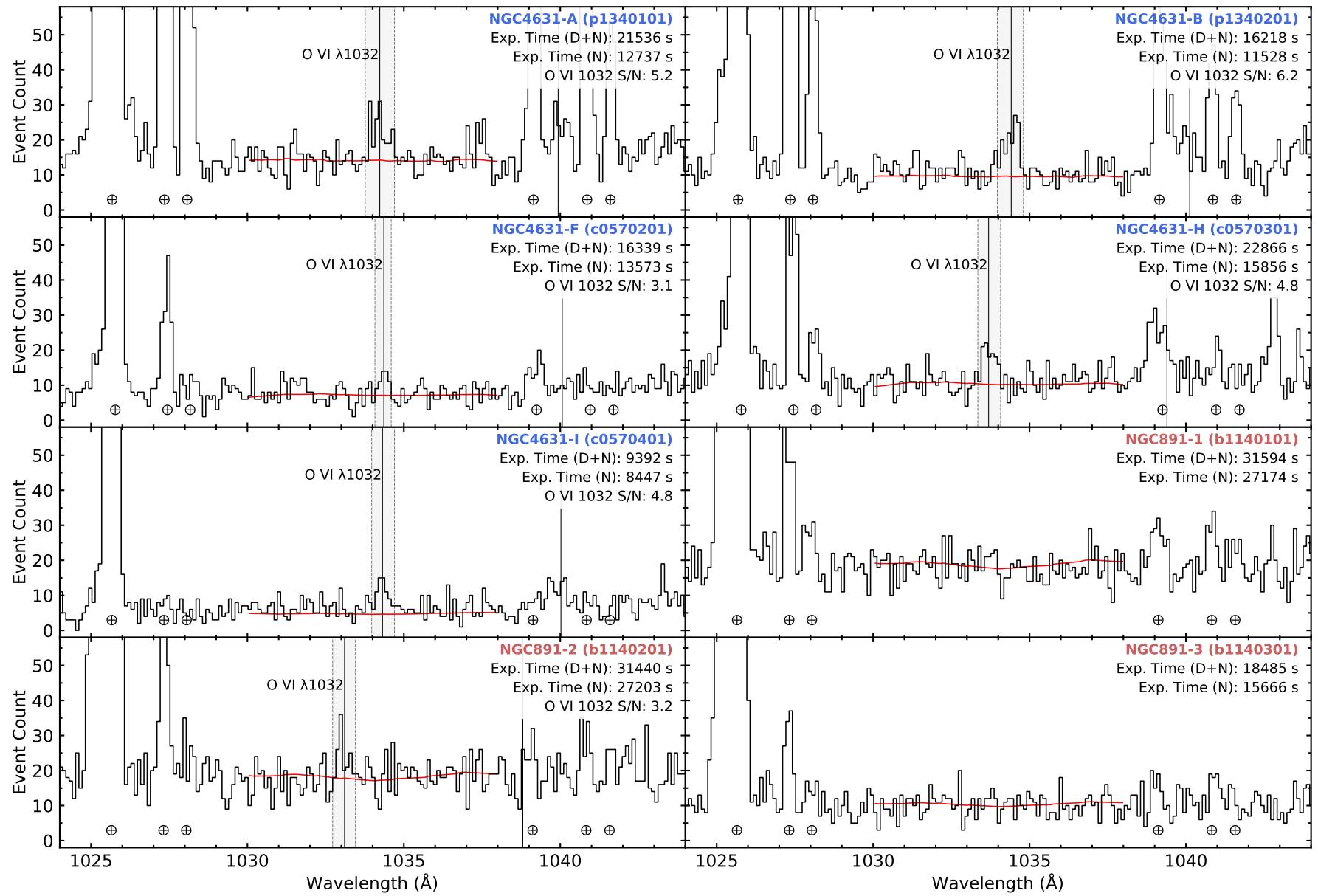

Figure 2. FUSE LWRS Day+Night spectra of NGC 4631-A, B, F, H, I fields and NGC 891-1, 2, 3 fields. The spectra were binned by 16 pixels. O VI $1032 \AA$ line centers derived from kinematic fitting are plotted as vertical black line, as well as the corresponding O VI $1038 \AA$ location. Background count spectrum around O VI $1032 \AA$ line is plotted in red. The width of the emission line extraction window is shown with the grey shaded area. Airglow line locations are marked with the Earth symbol. Exposure time (Day+Night and night only) and SNR of the O VI line emission are marked at each panel. Note that the background count spectrum is proportional to the exposure time.

\subsubsection{ROSAT data}

The NGC 4631 FUSE field points were chosen based on this ROSAT observation. Note that higher-resolution $\mathrm{X}$-ray data is available from more recent missions such as XMM-Newton or Chandra X-ray Observatory. Here we use only ROSAT data to show the correlation between the soft X-ray band contour (0.15-0.3 keV) and the FUSE field points. We re-analyzed the X-ray observation made by the ROSAT Position Sensitive Proportional Counter (PSPC, Truemper (1982)) on NGC 4631. The result was previously published by Wang et al. (1995).

Here we reproduce that result and show the soft $\mathrm{X}$ ray band contour in Figure 1. The ROSAT data of NGC 4631 (sequence id: rp600129a00, rp600129a01) is downloaded from the ROSAT Data Archive at Goddard Space Flight Center. First, we examined the event rate exten- sion (EVRATE) from the ancillary file (*_anc.fits) to manually define the good time intervals. This step is necessary to exclude bad time intervals contaminated by scattered solar X-rays. The remaining good time intervals are $14.2 \mathrm{ks}$ from rp600129a00 and $3.5 \mathrm{ks}$ from rp00129a01. With the remaining data (total $17.8 \mathrm{ks}$ ), we construct a 3D X-Ray data cube (X, Y, and pulse height) using the events list (STDEVT) from the basic file (*_bas.fits). The final soft-band X-ray image is made from the pulse height-invariant channels interval between 20 to 41, as described in Wang et al. (1995). This interval corresponds to the energy range $0.15-0.3$ $\mathrm{keV}$. The contour in Figure 1 is constructed with a pixel size of $5^{\prime \prime}$ and smoothed by a FWHM $=40^{\prime \prime}$ Gaussian.

\section{RESULT}

3.1. $N G C 4631$ 
Table 2. O VI Derived Parameters

\begin{tabular}{cccccccc}
\hline \hline Field & Program ID & $\begin{array}{c}\mathrm{z} \\
(\mathrm{kpc})\end{array}$ & $\begin{array}{c}\mathrm{I} 1032 \\
\left(\mathrm{cnts} / \mathrm{s} / \mathrm{cm}^{2} / \mathrm{sr}\right)\end{array}$ & $\begin{array}{c}\mathrm{I}_{1032}\left(\times 10^{-18}\right. \\
\left.\mathrm{ergs} / \mathrm{s} / \mathrm{cm}^{2} / \operatorname{arcsec}^{2}\right)\end{array}$ & $\begin{array}{c}\text { Signal, Background } \\
(\text { counts, counts })\end{array}$ & $\begin{array}{c}\text { Line Center } \\
(\AA)\end{array}$ & $\begin{array}{c}\text { Line FWHM } \\
(\AA)\end{array}$ \\
\hline NGC4631-A & $\mathrm{p} 1340101$ & 4.9 & $6000 \pm 1200$ & $2.7 \pm 0.5$ & 73,127 & $1034.23 \pm 0.09$ & $0.56 \pm 0.25$ \\
NGC4631-B & $\mathrm{p} 1340201$ & 2.7 & $8300 \pm 1300$ & $3.7 \pm 0.6$ & 76,76 & $1034.41 \pm 0.07$ & $0.41 \pm 0.19$ \\
NGC4631-F & $\mathrm{c} 0570201$ & 2.8 & $2900 \pm 1000$ & $1.3 \pm 0.4$ & 23,35 & $1034.36 \pm 0.08$ & $0.15 \pm 0.15$ \\
NGC4631-H & $\mathrm{c} 0570301$ & 2.4 & $4700 \pm 1000$ & $2.1 \pm 0.5$ & 53,71 & $1033.69 \pm 0.06$ & $0.34 \pm 0.12$ \\
NGC4631-I & $\mathrm{c} 0570401$ & 1.6 & $8600 \pm 1800$ & $3.9 \pm 0.8$ & 41,32 & $1034.32 \pm 0.07$ & $0.39 \pm 0.29$ \\
NGC891-1 & $\mathrm{b} 1140101$ & 2.0 & $<2200 a$ & $<1.0$ & & \\
NGC891-2 & $\mathrm{b} 1140201$ & 1.5 & $2300 \pm 700$ & $1.1 \pm 0.3$ & 40,124 & $1033.11 \pm 0.17$ \\
NGC891-3 & $\mathrm{b} 1140301$ & 4.1 & $<2700 a$ & $<1.2 a$ & & $0.36 \pm 0.49$ \\
\hline
\end{tabular}

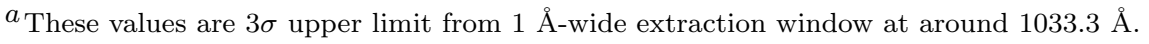

Figure 2 shows the resulting spectra (Day+Night) at the wavelength around O VI emission lines. The known airglow lines are visible in most of the spectra (H I 1025.7, OI 1025.8, 1027.4, 1028.2, 1039.2, 1040.9, 1041.7 $\AA$; Earth symbols in Figure 2). The O VI $1038 \AA$ line is not identifiable in most cases because it is blended with the airglow lines. However, the O VI $1032 \AA$ line is visible at the airglow-free window between 1030 to $1038 \AA$. Among the FUSE observations in Table 1, NGC 4631A* (Program ID: c0570101) is not presented because of the short exposure time, although the data shows an O VI $1032 \AA$ emission signal with $\mathrm{SNR}=2.7$. Another observation, NGC 4631-F* (Program ID: c0570202) is highly contaminated by unknown scattered light so the data is discarded from further analysis.

From the spectra, we derived O VI 1032 A intensity, signal counts, background counts, line center and line FWHM values. Those values are presented in Table 2. In this work, O VI $1032 \AA$ emission signal is detected from all five NGC 4631 fields, two (A, B) with SNR greater than 5, two $(\mathrm{H}, \mathrm{I})$ with $\mathrm{SNR}=4.8$, and the other $(\mathrm{F})$ with $\mathrm{SNR}=3.1$. We would like to note that the line detection at NGC 4631 field $\mathrm{F}$ is only a marginal detection. The derived O VI $1032 \AA$ intensity value at field A $\left(6000 \pm 1200\right.$ counts s$\left.{ }^{-1} \mathrm{~cm}^{-2} \mathrm{sr}^{-1}\right)$ and field $\mathrm{B}\left(8300 \pm 1300\right.$ counts $\left.\mathrm{s}^{-1} \mathrm{~cm}^{-2} \mathrm{sr}^{-1}\right)$ are higher than the ones reported by $\mathrm{O} 03\left(4600 \pm 1000\right.$ counts s${ }^{-1} \mathrm{~cm}^{-2}$ $\mathrm{sr}^{-1}$ for field $\mathrm{A}$ and $8000 \pm 1000$ counts s${ }^{-1} \mathrm{~cm}^{-2} \mathrm{sr}^{-1}$ for field B).

In particular, the signal from field $\mathrm{A}$ is estimated to be $30 \%$ higher than the result from O03. This is because of the differences between the data reduction pipeline used in this work (CalFUSE v3.2.3) and O03 (CalFUSE v2.0.5), such as pulse height range, extraction window height, good time interval definition, wavelength calibration, etc. Among those differences, the difference in the pulse height range is the main cause of the difference in
O VI intensity. O03 used a narrow pulse height range to reduce the background noise level. However, years later, it was reported that the use of a narrow pulse height range could result in flux losses (Dixon et al. 2007).

To verify whether the pulse height range is the major contributor of the difference, we have processed the raw data of NGC 4631 field A and B with the narrower pulse height range (4 and 15, inclusive, same as O03) using the latest pipeline (CalFUSE v3.2.3). The O VI 1032 $\AA$ intensity at the two fields are measured as $4800 \pm$ 1000 counts s${ }^{-1} \mathrm{~cm}^{-2} \mathrm{sr}^{-1}$ for field A and $8200 \pm 1300$ counts $\mathrm{s}^{-1} \mathrm{~cm}^{-2} \mathrm{sr}^{-1}$ for field $\mathrm{B}$, which are comparable to the previous estimation of $\mathrm{O} 03$.

The line center and line FWHM values of field A and field $\mathrm{B}$ agree with the result of O03. Note that the derived FWHM value assumed a fully illuminated LWRS $\left(30^{\prime \prime} \times 30^{\prime \prime}\right)$. Therefore the derived FWHM values can be considered as a lower limit.

\section{2. $N G C 891$}

Figure 2 shows the Day+Night spectra of three NGC 891 fields $(1,2,3)$ observed by FUSE LWRS. Previously all three fields were considered O VI $1032 \AA$ nondetections. In this re-visit of the FUSE spectra, we find a $3.2 \sigma$ signal of O VI $1032 \AA$ line emission at field 2 , although this is only a marginal detection. Field 1 and 3 still show non-detections but the lowest $3 \sigma$ upper limit $\left(<2200\right.$ counts $\mathrm{s}^{-1} \mathrm{~cm}^{-2} \mathrm{sr}^{-1}$, field 1$)$ is slightly increased compared to $\mathrm{O} 03\left(<2000\right.$ counts $\mathrm{s}^{-1} \mathrm{~cm}^{-2}$ $\mathrm{sr}^{-1}$, the same field 1 ).

The derived O VI $1032 \AA$ emission intensity, signal counts, background counts, line center and line FWHM values at field 2 are reported in Table 2 , along with the $3 \sigma$ upper limit intensity at field 1 and 3 . The line of sight velocity and line FWHM at field 2 are $342 \pm 51$ $\mathrm{km} / \mathrm{s}$ and $106 \pm 142 \mathrm{~km} / \mathrm{s}$, respectively.

\section{DISCUSSION AND CONCLUSIONS}


Here we discuss the potential implications of these O VI detections on our understanding of the distribution and kinematics of warm-hot coronal gas. We also discuss how this crucial component of galaxy halos can be best observed with future funded or proposed mission concepts.

\subsection{Implications for $O$ VI distribution}

The expected morphological distribution of O VI emitting warm-hot gas is debated. Simulations from Corlies \& Schiminovich (2016) show a filamentary and clumpy structure in the simulated O VI distribution that largely persists from $z=1$ to $z=0$ (e.g., Figure 6 , therein). The empirical work done by the COS-Halos survey in absorption line systems shows large covering fractions for O VI absorption within $75 \mathrm{kpc}$ of star-forming galaxies (Werk et al. 2013). This implies a more uniformly diffuse O VI morphology in actively star-forming galaxies, not the filaments show in simulations.

The vertical variation of intensity, velocity, and O VI FWHM for our sample fields are shown in Figure 3. The results of this study provide preliminary evidence for a filamentary or clumpy O VI morphological distribution in the inner CGM. NGC 4631 fields $\mathrm{F}$ and $\mathrm{H}$ show the weakest $\mathrm{O}$ VI emission, despite their proximity to the disk and an H I supershell (Rand \& van der Hulst 1993). This stands in contrast to the stronger detections along a nearly vertical line connecting NGC 4631 fields A, B, and I, which we assert provides evidence for a $\mathrm{O}$ VIemitting filament.

Filamentary structures may be superimposed within a diffuse O VI halo that is less-intensely emitting, and below the detection threshold for many of the fields presented in this study. Filaments would then be the interface of infalling flows with a diffuse halo of fountain gas primarily originating from the disk. This interpretation brings the results from this study, Corlies \& Schiminovich (2016), and Werk et al. (2013) into agreement. The simulated O VI halo in Figure 6 from Corlies \& Schiminovich (2016) would suggest this is the most likely scenario. Volume-filled mapping observations of O VI in emission are needed for a more definitive view on the morphology of warm-hot halos in nearby galaxies.

The filament structure along fields A, B, and I lies above a well-documented molecular gas outflow near the disk (Irwin et al. 2011; Rand 2000). The strength of the $\mathrm{O}$ VI detections are stronger along the central filament than near the periphery of the disk. This is potentially due to the outflowing gas near the central molecular outflow interacting with infalling material within the filament, heating the gas, and enhancing O VI emission. Alternatively, the giant magnetic rope features discov-

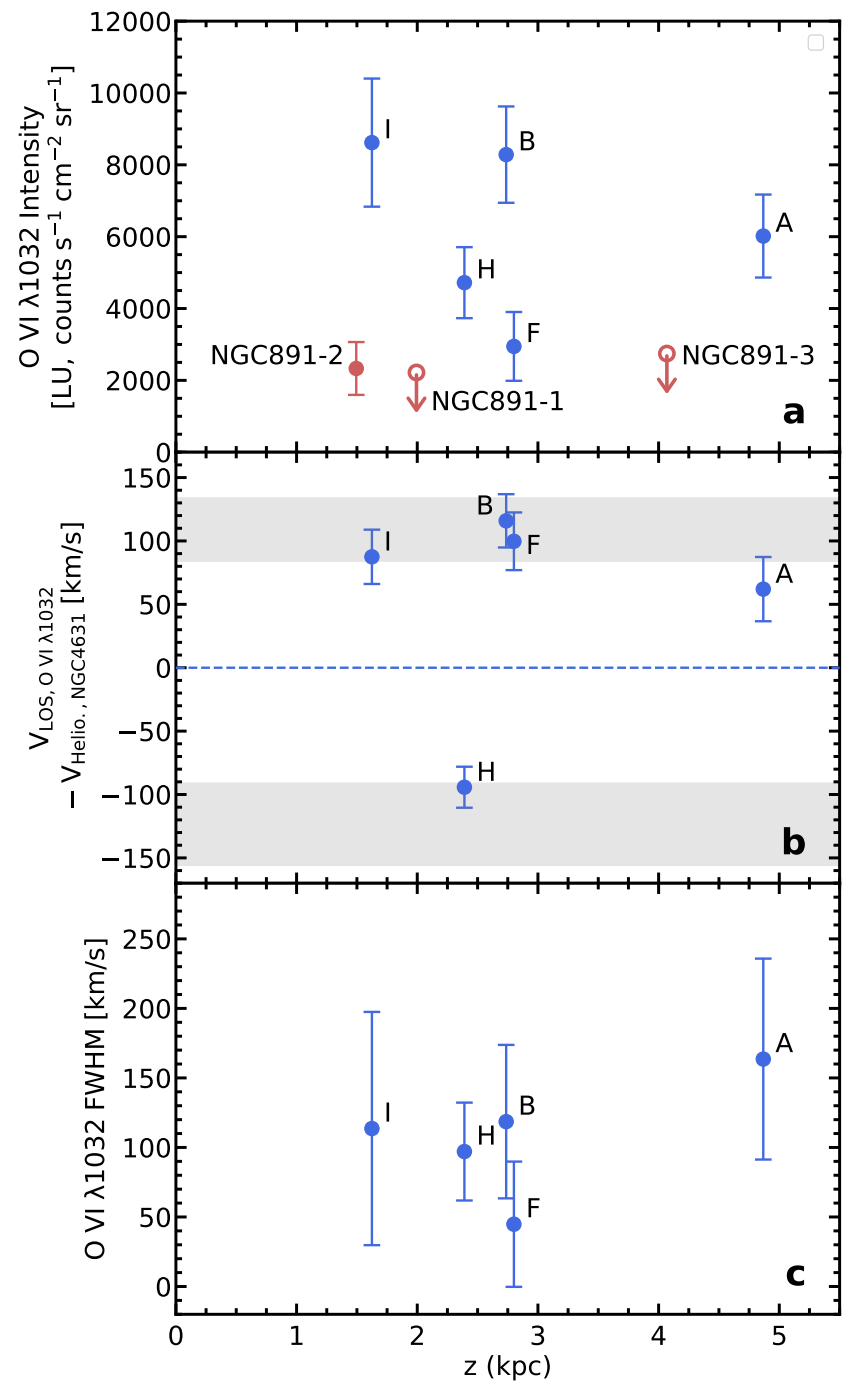

Figure 3. (a) O VI $1032 \AA$ line emission intensity, (b) lineof-sight velocity of O VI $1032 \AA$ with respect to the galaxy center $(606 \mathrm{~km} / \mathrm{s}$; Heliocentric velocity to NGC 4631, de Vaucouleurs et al. (1991)), and (c) FWHM of O VI $1032 \AA$, all as a function of the vertical height from the disk. All blue points are from the NGC 4631 fields. One red filled point is from NGC 891 field 2 and the other two red points indicate $3 \sigma$ upper limit at NGC 891 field 1 and 3. Horizontal gray bars are shown in panel (b) that represent the range in $\mathrm{H} \mathrm{I}$ velocity within the disk below the FUSE pointings in NGC 4631, as measured using HALOGAS data in Wu (2014).

ered in Mora-Partiarroyo et al. (2019) may be altering the distribution of, or otherwise interacting with, the ionized O VI-emitting gas.

We find stronger O VI emission in NGC 4631 than in NGC 891. It is possible that this discrepancy is due to the difference in the virial temperature of the two galaxies. The mass of NGC 4631 is closer to that of an $\mathrm{L}^{*}$ galaxy, and thus its virial temperature would be closer to the temperatures required for O VI emission. Higher 
mass systems, like NGC 891, may have a larger fraction of their CGM in the higher ionization 'hot' phase, probed by O VII and other X-ray metal lines.

\subsection{Implications for O VI kinematics}

Panel (b) of Figure 3 shows the vertical kinematic distribution of O VI emitting gas in each of the observed fields in NGC 4631, relative to the galaxy's systemic velocity. NGC 4631 field $\mathrm{H}$ is the only field on the rotationally approaching side of the galaxy, while the other fields are all on the receding side. The rotational direction of the underlying disk of NGC 4631 was checked using public $\mathrm{H}$ I data from the HALOGAS survey (Heald et al. 2011). The H I data verifies that the disk rotation matches the roation direction implied by each O VI detection above the disk in NGC 4631.

Though there are only five fields included, the vertical O VI kinematics can be compared to other inner-halo phase kinematics. Gas kinematics above spiral galaxy disks are expected to kinematically 'lag' the underlying disk rotation due to angular momentum conservation of ejected disk matter (Bregman 1980). Wu (2014) obtained vertical slit spectroscopy using the Apache Point Observatory (APO) 3.5-m Telescope to study the vertical kinematics of $\mathrm{H} \alpha$ emission in NGC 4631. The O VI filament along fields A, B, and I most closely correspond to slit 2 in the central field from $\mathrm{Wu}$ (2014). The $\mathrm{H} \alpha$ kinematics along that slit show roughly the same velocity magnitude as we find along fields A, B, and I (within uncertainties). However, for recently outflowing gas, one would expect the largest rotational velocity to be in the field closest to the disk - it will lag the disk rotation more intensely the further out it travels. It is not clear that that behavior is found along fields A, B, and I.

Fields $\mathrm{F}$ and $\mathrm{H}$, which lay further from the galaxy's center radially, both show velocities very close to the rotation curve at their radial distance, as inferred by an $\mathrm{H}$ I position-velocity diagram along the major axis (data from Heald et al. 2011; presented in Wu 2014). This regular rotation further supports the hypothesis that the O VI emitting gas from fields $\mathrm{F}$ and $\mathrm{H}$ represent originally shock-heated ejected matter that is cooling and falling down back onto the galaxy's disk. Since the velocity at points $\mathrm{F}$ and $\mathrm{H}$ do not show a lag to the underlying disk rotation (within uncertainties), this gas may be outflowing and recently ejected from the disk. We note that these inferences are made using a small number of available data points, and that a spectroscopic, volume-filled O VI emission mapping campaign is needed for more robust conclusions.

Panel (c) of Figure 3 includes the measured O VI FWHM values as a function of vertical distance. The

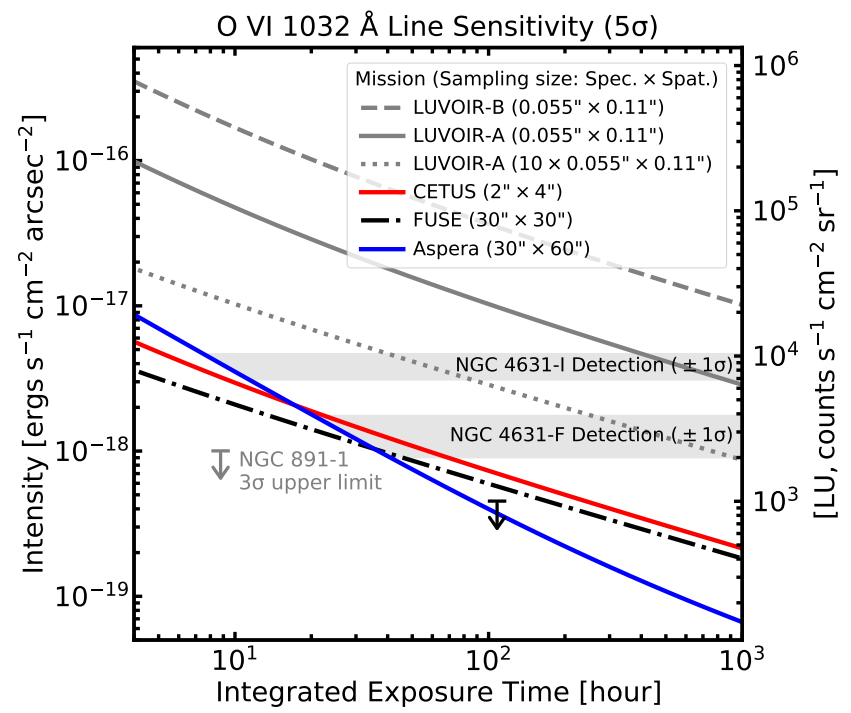

Figure 4. O VI $1032 \AA$ line emission sensitivity ( $5 \sigma$, per sampling element) of the past (FUSE) and future (LUVOIRA, LUVOIR-B, CETUS, and Aspera) UV missions. Each line is expected sensitivity vs. exposure time of each mission for a given sampling size with a specific configuration (Table B.1, Appendix B). Two of O VI emission detection ranges $( \pm 1 \sigma)$ and one non-detection upper limit $(3 \sigma)$ are also shown. The dotted grey line represents the sensitivity of LUVOIR-A with co-added O VI emission from ten $0.054^{\prime \prime}$ $\times 0.108^{\prime \prime}$ micro shutter apertures. The black arrow indicates the sensitivity requirement of Aspera mission at the exposure time of 4.5 days.

FWHM values are relatively uniform and show no clear trend or behavior.

\subsection{Implications for $O$ VI emission detection/mapping with future missions}

Studies of galaxy halos using HST-COS have yielded a significant amount of information and insight into the composition of various CGM components (Werk et al. 2013), including the seemingly large covering fraction of O VI. But the limitations of relying on infrequent background QSOs to probe foreground galaxy halos is clear with this work. The limited spatial coverage by FUSE of NGC 4631 and NGC 891 demonstrate that full coverage of a galaxy halo is needed to definitively conclude if there is a uniform or filamentary distribution of O VI gas. Here we briefly discuss the possibilities of doing wide-field, imaging spectroscopy over a full galaxy halo, comparing several proposed and funded mission concepts.

The signature of O VI is extremely faint and spread out over a large angular area for nearby galaxies (10s of arcmin), which motivates a wide field but high throughput telescope with moderate spectral resolution. Moderate spectral resolution will allow us to distinguish be- 
tween nearby geocoronal lines and identify the velocity shift of the O VI line. The FUSE telescope had relatively low throughput ( $\sim 2 \%$ end-to-end throughput), but a high enough effective area $\left(27 \mathrm{~cm}^{2}\right)$ to be sensitive to the detections described in this paper. For the detections described here, the dominant noise source was the FUSE detector background. The sensitivity of FUSE to the O VI line is shown in Figure 4, the black dash-dotted line, with the detection limits from NGC 4631 and NGC 891 shown.

We compare this performance with the performance of three proposed UV missions: LUVOIR-A, LUVOIRB (The LUVOIR Team 2019), and CETUS (Heap et al. 2019). LUVOIR (grey lines), despite its large aperture, has extremely small resolution elements from the microshutter array $\left(0.054^{\prime \prime} \times 0.108^{\prime \prime} ;\right.$ spectral $\times$ spatial $)$. This limits the ultimate amount of light collected in any one resolution element, which is challenging for very nearby targets and yields low sensitivity, even for very large apertures. Co-adding many micro-shutter elements together provides better sensitivity (dotted grey line shows 10 resolution elements binned together). CETUS (red line) performs very similarly to FUSE, per a $2^{\prime \prime} \times 4^{\prime \prime}$ $($ spectral $\times$ spatial) area on the slit. We put details of the sensitivity calculation in Appendix B.

We contrast these mission concepts with Aspera, a recently funded NASA Pioneer's SmallSat (PI: Dr. Carlos Vargas). Aspera was explicitly designed to observe $\mathrm{O}$ VI from nearby edge-on galaxies, and is able to reach a higher sensitivity than FUSE, LUVOIR, or CETUS per resolution element for exposures greater than $\sim 30$ hours, despite its small size and low cost. Aspera's design is directly inspired by FUSE and uses four off-axis parabola primary mirrors and Rowland circle spectrographs to achieve high throughput and moderate resolution while covering a large spatial area. Aspera is designed with a resolution element of $30^{\prime \prime} \times 60^{\prime \prime}$ (spectral $\times$ spatial) area on the slit. Aspera can simultaneously observe a total area of $2^{\prime} \times 45^{\prime}$ region, split into four $30^{\prime \prime} \times 45^{\prime}$ size slits. Aspera's spectral resolution is $\sim 2000$ at 1035 A.

\subsection{Conclusions}

In this paper, we analyze the archival FUSE spectra from the halo of two nearby star-forming, gas-rich, edgeon galaxies, NGC 4631 and NGC 891. We re-processed the raw FUSE data using the latest version of the FUSE data reduction pipeline. Among the five FUSE pointings on NGC 4631, we have found three new O VI emission detections and confirmed the two previously known detections. Among the three FUSE pointings on NGC 891, we have found one O VI emission detection, contrary to the previously reported non-detections on all three pointings. The intensity and spatial distribution of the NGC 4631 O VI detections cannot be explained with a simple diffuse halo model surrounding the galaxy. Our results imply that a discrete filamentary structure exists in the warm-hot phase above the central disk of NGC 4631, within a lower intensity uniformly diffuse, regularly rotating halo. The warm-hot gas detections closest to the disk's periphery of NGC 4631 most likely represent a diffuse component that originates from the disk. A weak O VI detection is also observed from one field in NGC 891.

Observations of $\mathrm{O}$ VI provide a window into an important CGM phase for galaxies. A well designed small telescope can provide better sensitivity in a shorter time than a very large telescope which is not optimized around this type of wide field, diffuse signal. In these cases, a specifically designed mission such as Aspera, funded via the NASA Pioneers Program, can answer extremely important science questions at a fraction of the cost of a Flagship mission.

\section{ACKNOWLEDGEMENT}

We thank the anonymous reviewer for their comments on this work.

\section{REFERENCES}

Behroozi, P., Wechsler, R. H., Hearin, A. P., \& Conroy, C. 2019, MNRAS, 488, 3143, doi: 10.1093/mnras/stz1182

Bregman, J. N. 1980, ApJ, 236, 577, doi: 10.1086/157776

Cheng, K. P., Collins, N., Angione, R., et al. 1997, UITVi; Vol. U

Corlies, L., \& Schiminovich, D. 2016, ApJ, 827, 148, doi: 10.3847/0004-637X/827/2/148

Das, S., Sardone, A., Leroy, A. K., et al. 2020, ApJ, 898, 15, doi: 10.3847/1538-4357/ab97b9 de Vaucouleurs, G., de Vaucouleurs, A., Corwin, Herold G.,

J., et al. 1991, Third Reference Catalogue of Bright

Galaxies

Dixon, W. V., Sahnow, D. J., Barrett, P. E., et al. 2007,

PASP, 119, 527, doi: 10.1086/518617 
France, K., Fleming, B., West, G., et al. 2017, in Society of Photo-Optical Instrumentation Engineers (SPIE) Conference Series, Vol. 10397, Society of Photo-Optical Instrumentation Engineers (SPIE) Conference Series, 1039713, doi: 10.1117/12.2272025

Grimes, J. P., Heckman, T., Strickland, D., et al. 2007, ApJ, 668, 891, doi: 10.1086/521353

Hayes, M., Melinder, J., Östlin, G., et al. 2016, ApJ, 828, 49, doi: 10.3847/0004-637X/828/1/49

Heald, G., Józsa, G., Serra, P., et al. 2011, A\&A, 526, A118, doi: 10.1051/0004-6361/201015938

Heap, S., Arenberg, J., Hull, T., Kendrick, S., \& Woodruff, R. 2019, arXiv e-prints, arXiv:1909.10437. https://arxiv.org/abs/1909.10437

Hodges-Kluck, E. J., Bregman, J. N., \& Li, J.-t. 2018, ApJ, 866, 126, doi: 10.3847/1538-4357/aae38a

Irwin, J. A., Wilson, C. D., Wiegert, T., et al. 2011, MNRAS, 410, 1423, doi: 10.1111/j.1365-2966.2010.17510.x

Kennicutt, Robert C., J., Armus, L., Bendo, G., et al. 2003, PASP, 115, 928, doi: 10.1086/376941

Moos, H. W., Cash, W. C., Cowie, L. L., et al. 2000, ApJL, 538, L1, doi: 10.1086/312795

Mora-Partiarroyo, S. C., Krause, M., Basu, A., et al. 2019, A\&A, 632, A11, doi: 10.1051/0004-6361/201935961

Murphy, E. M. 2002, Observations of O VI Emission in the Halo of NGC 4631, FUSE Proposal

Otte, B., Murphy, E. M., Howk, J. C., et al. 2003, ApJ, 591, 821, doi: 10.1086/375535

Pfeffermann, E., Briel, U. G., Hippmann, H., et al. 1987, in Society of Photo-Optical Instrumentation Engineers (SPIE) Conference Series, Vol. 733, Soft X-ray optics and technology, 519

Qu, Z., Bregman, J. N., \& Hodges-Kluck, E. J. 2019, ApJ, 876, 101, doi: 10.3847/1538-4357/ab17df
Radburn-Smith, D. J., de Jong, R. S., Seth, A. C., et al. 2011, ApJS, 195, 18, doi: 10.1088/0067-0049/195/2/18

Rand, R. J. 2000, ApJ, 535, 663, doi: 10.1086/308869

Rand, R. J., \& van der Hulst, J. M. 1993, AJ, 105, 2098, doi: 10.1086/116587

Richter, P., Winkel, B., Wakker, B. P., et al. 2018, ApJ, 868, 112, doi: 10.3847/1538-4357/aae838

Rogerson, J. B., Spitzer, L., Drake, J. F., et al. 1973, ApJL, 181, L97, doi: 10.1086/181194

Siegmund, O. H., Gummin, M. A., Stock, J. M., et al. 1997, in Society of Photo-Optical Instrumentation Engineers (SPIE) Conference Series, Vol. 3114, EUV, X-Ray, and Gamma-Ray Instrumentation for Astronomy VIII, ed. O. H. Siegmund \& M. A. Gummin, 283-294, doi: $10.1117 / 12.283775$

Siegmund, O. H. W., McPhate, J. B., Curtis, T., et al. 2020, in Society of Photo-Optical Instrumentation Engineers (SPIE) Conference Series, Vol. 11454, Society of Photo-Optical Instrumentation Engineers (SPIE) Conference Series, 114541H, doi: 10.1117/12.2561753

Temple, R. F., Raychaudhury, S., \& Stevens, I. R. 2005, MNRAS, 362, 581, doi: 10.1111/j.1365-2966.2005.09336.x

The LUVOIR Team. 2019, arXiv e-prints, arXiv:1912.06219. https://arxiv.org/abs/1912.06219

Truemper, J. 1982, Advances in Space Research, 2, 241, doi: 10.1016/0273-1177(82)90070-9

Tumlinson, J., Peeples, M. S., \& Werk, J. K. 2017, ARA\&A, 55, 389, doi: 10.1146/annurev-astro-091916-055240

Wang, Q. D., Walterbos, R. A. M., Steakley, M. F., Norman, C. A., \& Braun, R. 1995, ApJ, 439, 176, doi: 10.1086/175162

Werk, J. K., Prochaska, J. X., Thom, C., et al. 2013, ApJS, 204, 17, doi: 10.1088/0067-0049/204/2/17

Wu, C. 2014, Doctoral Dissertation, New Mexico State University

Zaritsky, D., \& Courtois, H. 2017, MNRAS, 465, 3724, doi: $10.1093 / \mathrm{mnras} / \mathrm{stw} 2922$ 


\section{APPENDIX}

\section{A. LINE FITTING}

It is important to carefully estimate the uncertainty of fitted parameter, when the fitting is attempted on the low signal to noise ratio data. To estimate the error of the fitted parameter, we created 1000 Poisson random noise added spectra, fit the line function to each spectrum, and take a mean and standard variation of parameters from the 1000 fittings. Although CalFUSE returns flux and error, generating random noise using Gaussian statistics is not advised because Poisson statistics cannot be approximated to Gaussian statistics when the signal is low. Therefore, we generated the random noise added spectra by using the COUNTS spectrum (Raw counts in extraction window, Table 4.8 of FUSE data handbook ${ }^{2}$ ). The Poisson noise added spectrum is further scaled by the ratio between COUNTS and WEIGHT spectrum and used as an input for the fitting. NOISE spectrum is determined by the square root of COUNTS spectrum scaled by the ratio between COUNTS and WEIGHT spectrum. As described in subsection 2.3, the line profile function determined by four parameters (Amplitude and the FWHM of the O VI emission model, a center of the convolved function, and a constant) is fitted to each random-noise added spectrum. The fitting range is limited to $1030 \AA$ to $1038 \AA$. For the line fitting, we assumed a flat background, represented by a constant as one of the four parameters. In Figure A.1, we put the distribution of 1000 line profiles fitted to the 1000 Poisson random noise added NGC 4631-A spectra. A line profile with the mean parameter values is plotted in black dashed line. The residual spectrum is shown in the bottom panel.

\section{B. SENSITIVITY CALCULATION}

We calculate diffuse O VI $1032 \AA$ emission line sensitivity of the past (FUSE) and future (LUVOIR-A, LUVOIR-B, CETUS, and Aspera) UV missions. The detector of all four missions is micro-channel plate (MCP). The lines are assumed to be slightly redshifted to $1035 \AA$. We have considered only two sources of noise for the calculation: target shot-noise and intrinsic MCP background noise, because those two are the dominant sources of noise at the wavelength around $1030 \AA$ A. Other sources of noise could be earthshine (not applicable for LUVOIR or CETUS), Zodiacal light, or instrument-specific scattered/stray light, but their con-

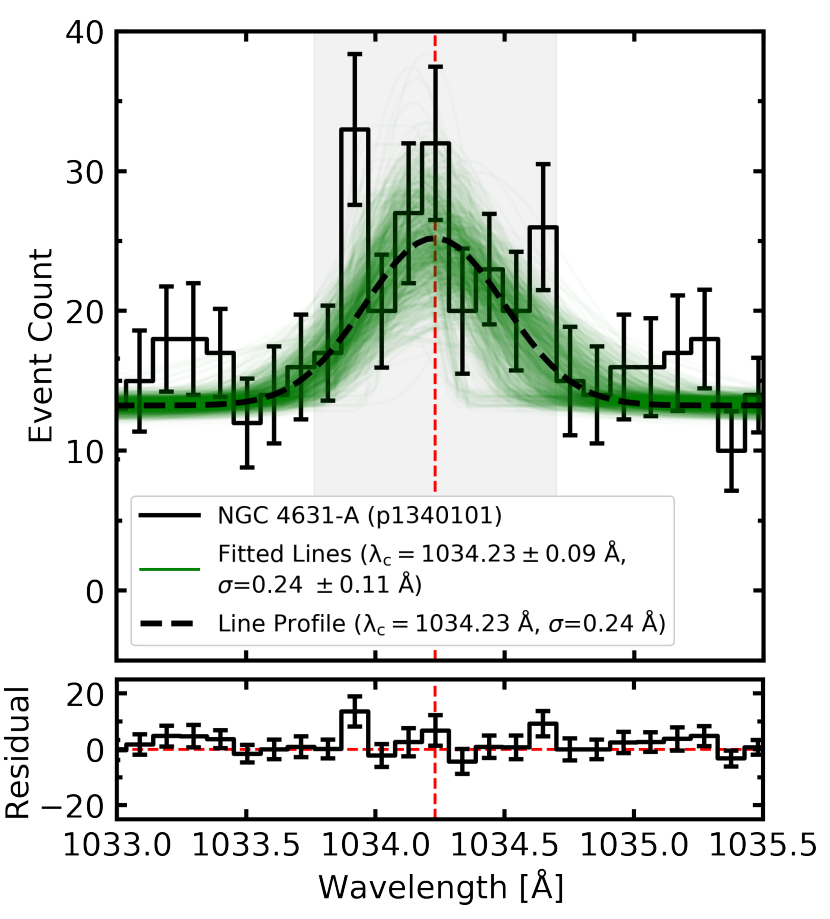

Figure A.1. (Top) The result of Monte-Carlo line-fitting to the O VI emission in NGC 4631-A spectrum. The spectrum is shown in black with $1 \sigma$ noise range. The 1000 overlaid green lines are line profiles fitted to the 1000 random Poissonnoise-added NGC 4631-A spectra. The black dashed line is the profile with mean line parameter values. Grey region indicates line emission extraction window. (Bottom) The residual spectrum between the NGC 4631-A spectrum and the line profile with the average parameter values.

tribution to the noise would be negligible at this wavelength range. Earthshine (assuming the Earth limb angle $>15^{\circ}$ ) and Zodiacal light are considerably dimmer than $10^{-21} \mathrm{ergs} \mathrm{s}^{-1} \mathrm{~cm}^{-2} \operatorname{arcsec}^{-2}$ (COS Instrument Handbook ${ }^{3}$ ). Scattered/stray light is assumed to be well-managed with quality optics, proper optomechanical arrangements and baffling. With the two kinds of noise sources, the sensitivity is calculated as a function of exposure time. The result is presented in Figure 4. Representative SNR breakdown is shown in Table B.1 for the case of LUVOIR-A, CETUS, FUSE, and Aspera at $1035 \AA$ with the exposure time of 388,000 sec (4.5 days, the nominal total exposure time per exposure of Aspera mission). The grasp (effective area

\footnotetext{
${ }^{3}$ https://hst-docs.stsci.edu/cosihb
}

2 https://archive.stsci.edu/fuse/dh.html 
Table B.1. SNR Calculation Breakdown per sampling element ${ }^{a}$ at $1035 \AA$ (Exposure time: 388800 sec)

\begin{tabular}{|c|c|c|c|c|c|}
\hline Parameters & $\begin{array}{c}\text { LUVOIR-A } \\
\text { (LUMOS, 155L) }\end{array}$ & $\begin{array}{c}\text { CETUS } \\
\text { (LUV G117) }\end{array}$ & $\begin{array}{c}\text { FUSE } \\
(\text { LWRS, LiF1-A) }\end{array}$ & Aspera & Unit \\
\hline \multirow{2}{*}{ O VI 1032 line intensity } & 21800 & 1550 & 1270 & 827 & counts $\mathrm{s}^{-1} \mathrm{~cm}^{-2} \mathrm{sr}^{-1}$ \\
\hline & $9.83 \mathrm{E}-18$ & $7.01 \mathrm{E}-19$ & $5.72 \mathrm{E}-19$ & $3.73 \mathrm{E}-19$ & $\operatorname{erg~s}^{-1} \mathrm{~cm}^{-2} \operatorname{arcsec}^{-2}$ \\
\hline Effective area & $7.1 \mathrm{E}+04$ & $1.1 \mathrm{E}+03$ & 27 & 2.1 & $\mathrm{~cm}^{2}$ \\
\hline Full slit length & 91 & 120 & 30 & $2700 \times 4^{b}$ & $\operatorname{arcsec}$ \\
\hline Spatial sampling & 0.110 & 4 & 30 & 60 & $\operatorname{arcsec}$ \\
\hline Slit width & 0.055 & 2 & 30 & 30 & $\operatorname{arcsec}$ \\
\hline Total background rate at MCP & 1.05 & 0.30 & 0.96 & 0.30 & counts s${ }^{-1} \mathrm{~cm}^{-2}$ \\
\hline Extraction aperture size (Spatial) & 0.0159 & 0.0508 & 0.0800 & 0.0047 & $\mathrm{~cm}$ \\
\hline Extraction aperture size (Spectral) & 0.0292 & 0.0844 & 0.0960 & 0.0087 & $\mathrm{~cm}$ \\
\hline Total signal count & 82 & 125 & 281 & 29 & counts \\
\hline Noise (MCP Background) & 14 & 22 & 54 & 2 & counts \\
\hline Noise (Total RSS) & 16 & 25 & 56 & 6 & counts \\
\hline Total Signal to Noise Ratio & 5.0 & 5.0 & 5.0 & 5.0 & \\
\hline Grasp $c$ & 98.4 & 73.3 & 6.7 & 192.6 & $\mathrm{~cm}^{2} \operatorname{arcmin}^{2}$ \\
\hline
\end{tabular}

${ }^{a}$ Spatial sampling $\times$ slit width

$b^{b}$ Four $45^{\prime} \times 30^{\prime \prime}$ slits

${ }^{c}$ Effective area $\times$ full slit length $\times$ slit width

$\times$ field-of-view) of the four missions are also shown in Table B.1.

Below we describe the justification of adopted parameters. The resulting sensitivity curves are the best estimates based on the available information and will be different if there are changes to the parameter values. As guidance for the impact of changes to the sensitivity, we have checked the sensitivity curve of the missions with two additional cases, (1) $20 \%$ larger effective area with $20 \%$ smaller background noise and (2) $20 \%$ smaller effective area with $20 \%$ larger background noise with respect to the baseline values. The sensitivity will be increased by $0.09-0.13$ dex for case (1), and decreased by $0.11-0.13$ dex for case (2).

\section{B.1. LUVOIR}

The instrument parameters of LUVOIR-A and B are estimated from the LUVOIR mission concept study final report (The LUVOIR Team 2019). LUMOS-G155L grating is chosen because the grating is expected to have the highest sensitivity to the O VI emission while provide a reasonable diffuse-source spectral resolution ( $\mathrm{R} \sim 2500)$, assuming a fully-illuminated micro shutter $\left(0.110^{\prime \prime} \times 0.055^{\prime \prime}\right)$. LUMOS can open 840 micro shutters simultaneously within the $2^{\prime} \times 2^{\prime}$ micro shutter array field of view. The effective area of G155L grating at 1035 $\AA$ is estimated by assuming the spectral shape of the effective area curve from Figure 8 of France et al. (2017), scaled by the peak effective area value of G155L grating from Table 8-9 of the LUVOIR final report (The LUVOIR Team 2019) for both LUVOIR-A and LUVOIR$\mathrm{B}$ concepts. MCP background rate of 1.05 counts $\mathrm{s}^{-1}$ $\mathrm{cm}^{-2}$ is taken from France et al. (2017). The extraction aperture size $(293 \mu \mathrm{m} \times 159 \mu \mathrm{m})$ corresponds to the size of fully-illuminated un-vignetted open area of a single microlens shutter size (corresponds to $0.054^{\prime \prime} \times$ $0.108^{\prime \prime}$ are on sky, physically $\left.79 \mu \mathrm{m} \times 159 \mu \mathrm{m}\right)$ with $1 \AA$ width on the detector. This assumes unit magnification of LUMOS FUV G155L optics.

\section{B.2. CETUS}

CETUS instrument parameter values are adopted from CETUS final report (Heap et al. 2019). Point/Slit spectrograph (PSS) with LUV G117 grating mode is chosen because this is the only available CETUS configuration to observe local O VI $1032 \AA$ emission line. The effective area of CETUS G117 configuration at $1035 \AA$ is estimated from Figure 1-3 of Heap et al. (2019). MCP background rate is assumed as 0.3 counts $\mathrm{s}^{-1} \mathrm{~cm}^{-2}$. The MCP background rate is estimated by the summation of the atomic layer deposited (ALD) MCP background rate measured at the ground $\left(0.05\right.$ counts $\left.\mathrm{s}^{-1} \mathrm{~cm}^{-2}\right)$ and the expected difference between the flight and ground background rate with ALD MCP with satellite mass of $\sim 2.6$ metric ton $\left(0.25\right.$ counts s${ }^{-1} \mathrm{~cm}^{-2}$, Siegmund et al. (2020)). The extraction aperture size on the detector $(844 \mu \mathrm{m} \times 508 \mu \mathrm{m})$ corresponds to the size of O VI emission with $1 \AA$ width, fully-illuminated $2^{\prime \prime} \times 4^{\prime \prime}$ (spectral 
$\times$ spatial) area on the slit. 1:2 ratio of the sampling element size is chosen arbitrarily to be aligned with the sampling shape of LUVOIR micro shutter. The length of CETUS PSS LUV slit is $2^{\prime}$, so 30 such sampling elements can be observed simultaneously.

\section{B.3. FUSE}

Although FUSE is already retired, an estimation with FUSE is also shown as a reference. Here we took the instrument parameters as per the NGC 4631-A observation (Effective area $=26.9 \mathrm{~cm}^{2}$, Total background rate $=0.96$ counts $\left.\mathrm{s}^{-1} \mathrm{~cm}^{-2}\right)$ and the FUSE instrument handbook $^{4}$. The field of view of LWRS is $30^{\prime \prime} \times 30^{\prime \prime}$. The extraction aperture size corresponds to the footprint of fully illuminated LWRS on the detector at around $1035 \AA$ (Height: $800 \mu \mathrm{m}$; identified from detector image, width: $960 \mu \mathrm{m}$; corresponds to $1.08 \AA$ ).

\section{B.4. Aspera}

Aspera instrument parameters are adopted from Aspera proposal that was submitted to 2020 NASA Pioneers Announcement of Opportunity (Vargas et al. Private Comm.). Effective area of $2.1 \mathrm{~cm}^{2}$ at $1035 \AA$ and total background rate of 0.3 counts $\mathrm{s}^{-1} \mathrm{~cm}^{-2}$ are used for the sensitivity calculation. The extraction aperture size $(47 \mu \mathrm{m} \times 87 \mu \mathrm{m})$ on the detector corresponds to the size of $1 \AA$ width O VI emission with fully-illuminated $30^{\prime \prime} \times 60^{\prime \prime}($ spectral $\times$ spatial $)$ area on the slit. By employing the step-and-stare concept observation, Aspera can effectively map the spatial distribution of O VI emission in galaxy halos.

${ }^{4}$ https://archive.stsci.edu/fuse/ih.html 